\title{
Identifying Training Requirements in Perioperative Care for Anaesthetists
}

\author{
Gautam Kumar, ${ }^{1}$ Beverly Wong, ${ }^{2}$ and David Walker ${ }^{2}$ \\ ${ }^{1}$ University College London (UCL), Division of Surgery, Rockefeller Building, London WC1E 6BT, UK \\ ${ }^{2}$ Centre for Anaesthesia, University College London Hospitals, 235 Euston Road, London NW1 2BU, UK \\ Correspondence should be addressed to Gautam Kumar; gautamkumar@doctors.org.uk
}

Received 22 August 2013; Revised 12 November 2013; Accepted 13 November 2013

Academic Editor: Stephen D. Helmer

Copyright (c) 2013 Gautam Kumar et al. This is an open access article distributed under the Creative Commons Attribution License, which permits unrestricted use, distribution, and reproduction in any medium, provided the original work is properly cited.

\begin{abstract}
Education and training in anaesthesia have traditionally focused on the preparation and delivery of intraoperative anaesthesia but are evolving to incorporate aspects of perioperative medical care. The expansion of continued professional development and postgraduate courses in this field has gathered pace, with the aim of teaching anaesthetists and allied professionals to improve patients' surgical care. We surveyed a population of UK-based anaesthetists to establish their views on professional development within perioperative medicine, their role as perioperative medical experts of the future, and the training and educational needs of this cohort. The majority of anaesthetists acknowledged their evolving role in perioperative patient care and recognised a need to train for the task. Only $50 \%$ of the senior anaesthetists surveyed believed they had sufficient knowledge and skills to undertake perioperative care with the majority believing the current training curriculum must advance to support professional development. In line with other international healthcare systems, UK-based anaesthetic practice is adopting a responsibility for perioperative medical practice, and this survey has demonstrated willingness amongst anaesthetists of all grades to embrace change, recognise training needs, and improve the outcome for surgical patients.
\end{abstract}

\section{Introduction}

A growing proportion of patients undergoing surgery are elderly and increasingly have coexistent disease, which may influence outcome $[1,2]$. As surgical programmes develop to meet the needs of this population, it is now commonplace to see this cohort undergo major surgical interventions, which expose them to a high possibility of perioperative morbidity and mortality [3]. Such patients are often cared for within resource-limited healthcare systems, whose continued remits are to drive efficiency and streamline clinical episodes and may be at odds with best care for those patients considered to be at highest risk. This so-called high-risk patient population represents approximately $15 \%$ of the total surgical population and yet as a group contributes to $80 \%$ of perioperative mortality [4]. Current models of care, involving surgical teams working without the support of multidisciplinary input, have been challenged and led to calls from within the profession to develop broad-based perioperative medicine expertise, which better supports complex patient episodes.
Perioperative medicine is defined as "the continuum of patient care involving preoperative evaluation and preparation, preanaesthetic assessment, intraoperative care, and the management of systems and personnel supporting these activities" [5]. Clearly, this broader remit for patient care challenges the traditional role of anaesthetic practice and might be considered an opportunity for professional development and even a paradigm shift in the delivery of care. In the UK, a survey of practising anaesthetists highlighted that they were working in the operating theatre for only $50 \%$ of their time and spending $10 \%$ of their time based in a ward environment [6]. This survey, now more than a decade old, signaled the change that was beginning to occur from theatre-based practitioners to perioperative physicians. Most recently, a statement by the President of the Royal College of Anaesthetists in November 2013 stated "one of the strategic aims of the college for 2013-2014 is to explore the role anaesthetists might play in the delivery of perioperative medicine and how we might develop a more formal approach to meeting this agenda" [7]. Against the backdrop of this changing landscape, 
TABLE 1: Response rates by consultant and trainee anaesthetists to the quantitative questions in the survey.

\begin{tabular}{|c|c|c|c|c|c|c|}
\hline \multirow{2}{*}{ Survey questions } & \multicolumn{3}{|c|}{ Consultants } & \multicolumn{3}{|c|}{ Trainees } \\
\hline & Yes & No & Maybe & Yes & No & Maybe \\
\hline Should anaesthetists have a role in pre- and post-operative patient management? & 43 & 0 & 1 & 40 & 0 & 0 \\
\hline In the future, will anaesthetists have more responsibility in perioperative care? & 43 & 0 & 0 & 37 & 1 & 0 \\
\hline Does the FRCA syllabus adequately cover perioperative medicine? & 5 & 31 & 8 & 8 & 20 & 10 \\
\hline Have you sufficient training to become a perioperative physician? & 22 & 20 & 2 & 9 & 27 & 2 \\
\hline Would you be interested in a formal qualification in perioperative medicine? & 20 & 16 & 8 & 24 & 8 & 8 \\
\hline Would you be interested in an online course delivered by experts? & 37 & 3 & 4 & 30 & 6 & 4 \\
\hline
\end{tabular}

we conducted a structured survey exploring the professional attitudes, educational beliefs, and the training requirements of both trainee and consultant grade anaesthetists currently practicing in the UK.

\section{Methods}

A snapshot survey was conducted, comprising of 100 unselected anaesthetists of all grades in two large teaching hospitals as well as those attending an unrelated national intensive care meeting between October and November 2012. Anonymised questionnaires were designed to answer questions relating to perception of the current and future role of the UK-based anaesthetist, questions relating to training and the desire to become better trained in the area of perioperative medicine. Questionnaires were returned and collated by an independent researcher. Specifically, eight questions investigated anaesthetists' opinions of the major issues in perioperative medicine, whether they thought they were responsible for patients' perioperative care and whether, in the future, anaesthesia would incorporate a more diverse perioperative role. The survey also investigated anaesthetic education and questioned whether respondents had the appropriate training to become perioperative physicians. Six questions evaluated quantitative data and are displayed in Table 1 with the remaining two questions addressing qualitative opinions and ideas. These two open-ended questions were as follows.

(1) "What are the perioperative medical issues encountered that affect your clinical practice?"

(2) "If you were to undergo further education and training in perioperative medicine, which topics would you want to be included in the course syllabus?"

\section{Results}

Eighty-four percent of questionnaires were returned for analysis $(n=84)$ which were considered to be a suitable sample size with adequate response rates. Forty-four respondents were consultant anaesthetists and 40 respondents were trainees. Table 1 shows the quantitative results.

Ninety-nine percent of anaesthetists agreed that they have a role in pre- and postoperative patient management, and all but one person surveyed saw anaesthetists taking on more responsibility for patients' perioperative care in the future in addition to their traditional anaesthetic duties. Perhaps most interestingly, only $50 \%$ of consultants felt they have sufficient training to do this. With regard to this training in the UK, there is disparity with the fact that $62 \%$ of surveyed anaesthetists believe the FRCA syllabus does not adequately cover training in perioperative medicine. To resolve this, $45 \%$ of consultants and $60 \%$ of trainees welcomed further professional development in perioperative medicine.

Responding to the open-ended questions, anaesthetists identified that the major issues in perioperative medicine affecting their work include risk stratification and clinical management, patient optimisation and appropriate and effective preoperative assessment with over $50 \%$ of those surveyed making reference to these points. Other themes that arose included the management of complex cardiac and respiratory comorbidities, perioperative fluid balance, goal-directed therapy, postoperative ward care, inconsistent access to high-dependency facilities, and the difficulty of implementing enhanced-recovery programmes.

The second open-ended question showed that the most popular topics anaesthetists would want to learn about in a perioperative course include how to risk assess a patient preoperatively (involving the benefit of investigations, cardiopulmonary exercise testing, pulmonary function testing, etc.), the management of acute and chronic complex comorbidities, evidenced-based perioperative practice, enhanced-recovery and perioperative fluid management.

\section{Discussion}

A well-motivated anaesthetic professional is ideally suited to provide added value to the perioperative care of surgical patients. Anaesthetists practising perioperative medicine may be best able to integrate preoperative evaluation, risk assessment, optimisation of comorbidities, state-of-the-art intraoperative monitoring, and appropriate management of postoperative pain. It is believed that attention to these key components of perioperative care may positively affect morbidity and mortality, length of hospital stay, and patient satisfaction [8].

Our survey demonstrates an understanding within the anaesthetic community of the evolution in professional practice. When questioned about their future involvement in pre- and postoperative patient care, we observed overwhelming agreement from anaesthetists that they should have a more developed and expanded role. Importantly, respondents believed it is the duty of the anaesthetist rather than another group of health professionals to be involved in this additional 
care. It was acknowledged that anaesthetists already have many of the required skills to lead the perioperative medicine team. Overall, a minority of respondents believed they had sufficient training to perform this role adequately with a tendency to believe the Royal College of Anaesthetists' syllabus currently does not adequately support perioperative medical practice.

At present, the mortality rate for patients undergoing inpatient noncardiac surgery is higher than anticipated with variations in mortality between countries, suggesting the need for national and international strategies to improve care for this group of patients [9]. The expanded role of the anaesthetist in perioperative practice is one such strategy. Development of this standard of care in the UK appears to be following other developed countries. In 2005, the Australian and New Zealand College of Anaesthetists' (ANZCA) Perioperative Medicine Taskforce stated that a Perioperative Medicine module for trainees should be a compulsory core component of the curriculum and that there should be an establishment of opportunities for fellows to consolidate their knowledge in this area. It also stated that best practice implementation and outcomes-based research in perioperative medicine should be actively supported [10].

Similarly in the USA, the concept of the anaesthetist as the perioperative physician is strongly supported by the American Board of Anesthesiology (ABA), which in 2008 introduced changes in residency training to significantly increase experience in perioperative medicine. Their evolution process began slowly but discussions about change in focus began as long as 20 years ago with Greene and Saidman using the American Society of Anesthesiologists (ASA) annual Rovenstine lectures to recommend the reorientation of anaesthesia as "perioperative medicine and pain management" [11] to reflect such a new forward-thinking approach. However, anaesthesiologists in the USA have to contend with physicians known as hospitalists, who have established a role as perioperative physicians. Most hospitalists are trained in internal medicine and its subspecialties. The Hospitalists fulfil a role by caring for patients in the preoperative and sometimes in the postoperative period, allowing the surgeon to focus on surgical issues. These roles of the anesthesiologists and the hospitalists, as "Perioperative Physicians," may be complementary. It has been concluded that if anesthesiologists and hospitalists work together alongside surgeons in the shared care model of perioperative medical practice with each specialty bringing its expertise to the care of the perioperative patient, care is likely to improve [12].

To date, the majority of anaesthetic training and practice involves the preparation for and provision of intraoperative anaesthesia. This emphasis arose out of a need to make the operating room safer and improvements continue to be made $[13,14]$. The incidence of perioperative mortality that is directly attributable to anaesthesia had been quoted as much as 1 in 6,795 to 1 in 200,000 a decade ago [15] but a more recent study now reports this is drastically improving with a much lower mortality rate of 1.4 per million operations [16]. In the UK, the emergence of the physician's assistant grade in anaesthesia enables safe anaesthetic episodes to be delivered by nonphysician anaesthetists and is increasingly being recognised as making a significant contribution to the delivery of UK anaesthesia [17]. Such developments, when responsibly introduced, will allow clinician-anaesthetists time to focus their expertise on the high-risk patient population in both the operating room and expanding their practice to incorporate perioperative management [12].

Notwithstanding the demonstrated perceived need for higher training in the UK, our survey suggests that a significant proportion of consultants consider themselves appropriately trained in most aspects of perioperative medicine. Those not so confident would be prepared to undertake further education to improve their care and even work towards a higher professional qualification with greater enthusiasm, perhaps unsurprisingly, coming from those doctors in training. There seems to be considerable interest within the profession from both consultants and trainees to undertake such learning as an online, distant-learning course, particularly where learning materials could be delivered by experts. The use of Web-based education may be seen to improve the quality of life-long learning programmes and is seen as a modern, successful, educational medium, especially for doctors who may have several other professional commitments and would welcome a flexible approach to education [18].

\section{Conclusion}

The need to improve perioperative care for surgical patients is evident. Striking variations in patient outcome between institutions and nations continue to exist, and in one study, the overall care was rated as "good" in only $48 \%$ of high risk patients [19-21]. We believe that further training leading to skills in risk reduction, patient optimization, and grounding in perioperative medicine would reduce the disparity and result in better patient outcomes. We further believe that the well-trained anaesthetist is best placed to deliver such quality improvement and should now be supported by professional organisations to emerge from the operating theatre and lead the change.

\section{References}

[1] L. A. Anderson, R. A. Goodman, D. Holtzman, S. F. Posner, and M. E. Northridge, "Aging in the United States: opportunities and challenges for public health," The American Journal of Public Health, vol. 102, no. 3, pp. 393-395, 2012.

[2] R. Beaglehole, S. Ebrahim, S. Reddy, J. Voûte, and S. Leeder, "Prevention of chronic diseases: a call to action," The Lancet, vol. 370, no. 9605, pp. 2152-2157, 2007.

[3] R. M. Pearse, D. A. Harrison, P. James et al., "Identification and characterisation of the high-risk surgical population in the United Kingdom," Critical Care, vol. 10, no. 3, article R81, 2006.

[4] D. A. Story, K. Leslie, P. S. Myles et al., "Complications and mortality in older surgical patients in Australia and New Zealand (the REASON study): a multicentre, prospective, observational study," Anaesthesia, vol. 65, no. 10, pp. 1022-1030, 2010.

[5] V. Beavis, "Anaesthetists as perioperative physicians," in Proceedings of the Australian Society of Anaesthetists National Scientific Conference, 2012. 
[6] S. Ingram, "The Royal College of Anaesthetists," Bulletin, vol. 1, pp. 9-11, 2002.

[7] J. van Besouw, "The Royal College of Anaesthetists," Bulletin, vol. 82, pp. 4-5, 2013.

[8] C. Beattie, "Training perioperative physicians," Anesthesiology Clinics of North America, vol. 18, no. 3, pp. 515-525, 2000.

[9] R. M. Pearse, R. P. Moreno, P. Bauer et al., "Mortality after surgery in Europe: a 7 day cohort study.," The Lancet, vol. 380, no. 9847, pp. 1059-1065, 2012.

[10] Australian and New Zealand College of Anaesthetists, "Perioperative Medicine Taskforce Report," 2005.

[11] L. J. Saidman, "The 33rd Rovenstine Lecture: what I have learned from 9 years and 9,000 papers," Anesthesiology, vol. 83, no. 1, pp. 191-197, 1995.

[12] A. O. Adesanya and G. P. Joshi, "Hospitalists and anesthesiologists as perioperative physicians: are their roles complementary?" Proceedings, vol. 20, no. 2, article 140, 2007.

[13] C. S. Deutschman and K. B. Traber, "Evolution of anesthesiology," Anesthesiology, vol. 85, no. 1, pp. 1-3, 1996.

[14] M. A. Warner, S. E. Shields, and C. G. Chute, "Major morbidity and mortality within 1 month of ambulatory surgery and anesthesia," Journal of the American Medical Association, vol. 270, no. 12, pp. 1437-1441, 1993.

[15] R. S. Lagasse, "Anesthesia safety: model or myth? A review of the published literature and analysis of current original data," Anesthesiology, vol. 97, no. 6, pp. 1609-1617, 2002.

[16] P. J. Nkansah, D. A. Haas, and M. A. Saso, "Mortality incidence in outpatient anesthesia for dentistry in Ontario," Oral Surgery, Oral Medicine, Oral Pathology, Oral Radiology, and Endodontics, vol. 83, no. 6, pp. 646-651, 1997.

[17] AAGBI, "Physicians' Assistant (Anaesthesia) Review 2011," http://www.aagbi.org/sites/default/files/PA(A)\%20Review_FINAL\%2016MAR2012.pdf.

[18] M. Sajeva and V. Lanza, "Open Source Web based solutions for lifelong learning," in Proceedings of the 12th International Conference NETTIES, Editura Politehnica, Timisoara, Romania, 2006.

[19] M. P. W. Grocott and R. M. Pearse, "Perioperative medicine: the future of anaesthesia?" British Journal of Anaesthesia, vol. 108, no. 5, pp. 723-726, 2012.

[20] National Confidential Enquiry into Patient Outcome and Death and G. P. Findley, "Knowing the risk: a review of the perioperative care of surgical patients," NCEPOD, 2011.

[21] J. B. Dahl and H. Kehlet, "Perioperative medicine: a new subspeciality, or a multi-disciplinary strategy to improve perioperative management and outcome?" Acta Anaesthesiologica Scandinavica, vol. 46, no. 2, pp. 121-122, 2002. 

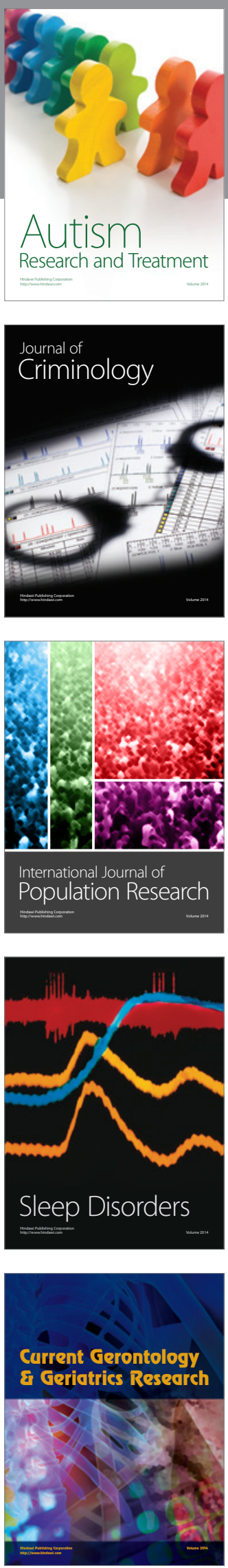
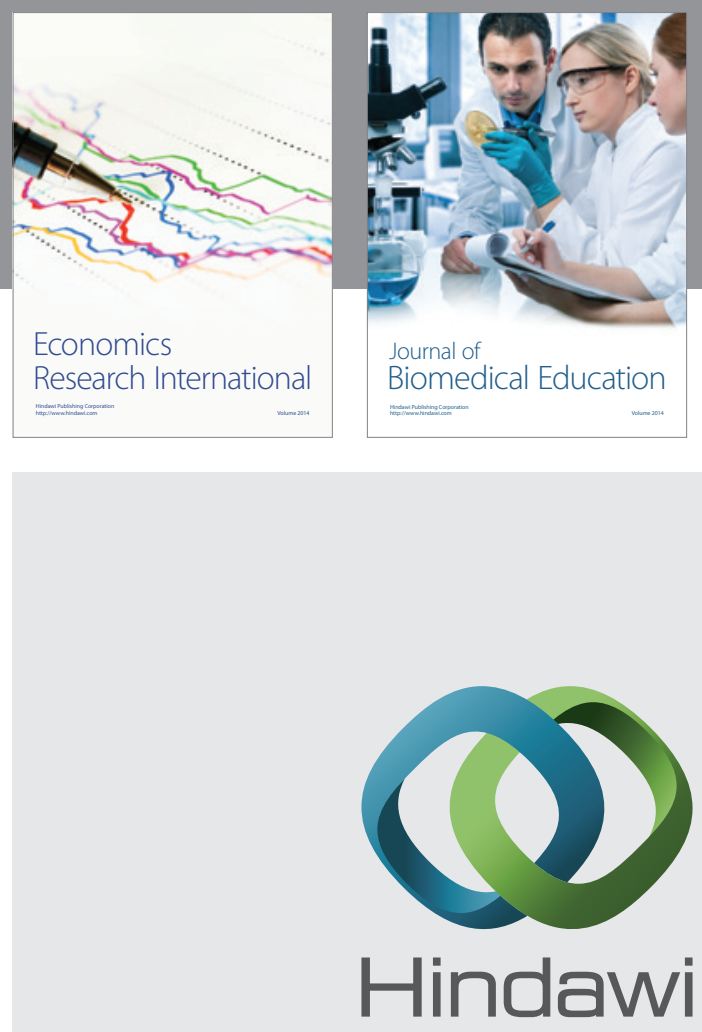

Submit your manuscripts at

http://www.hindawi.com
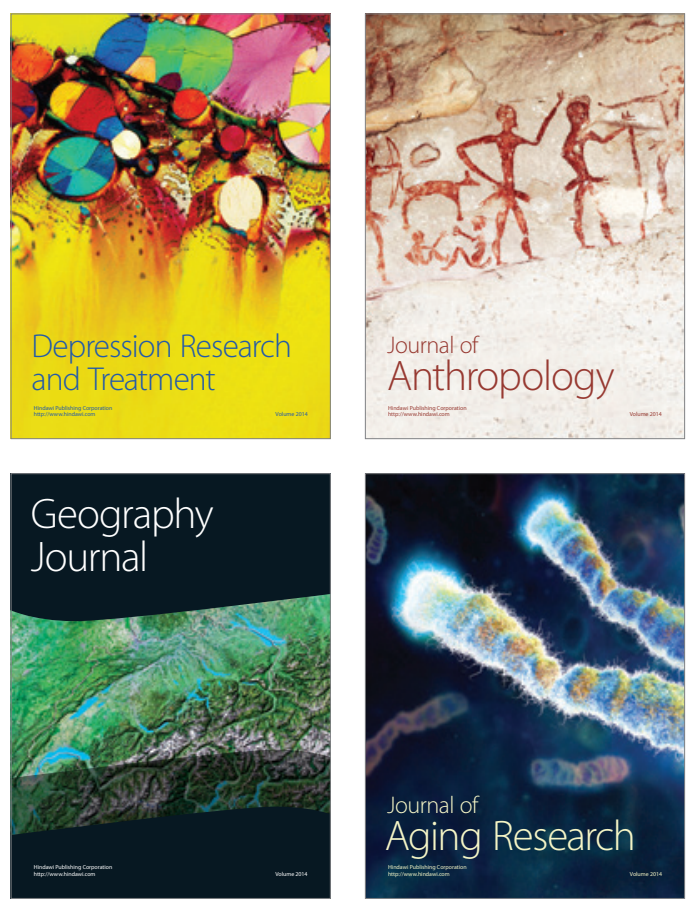
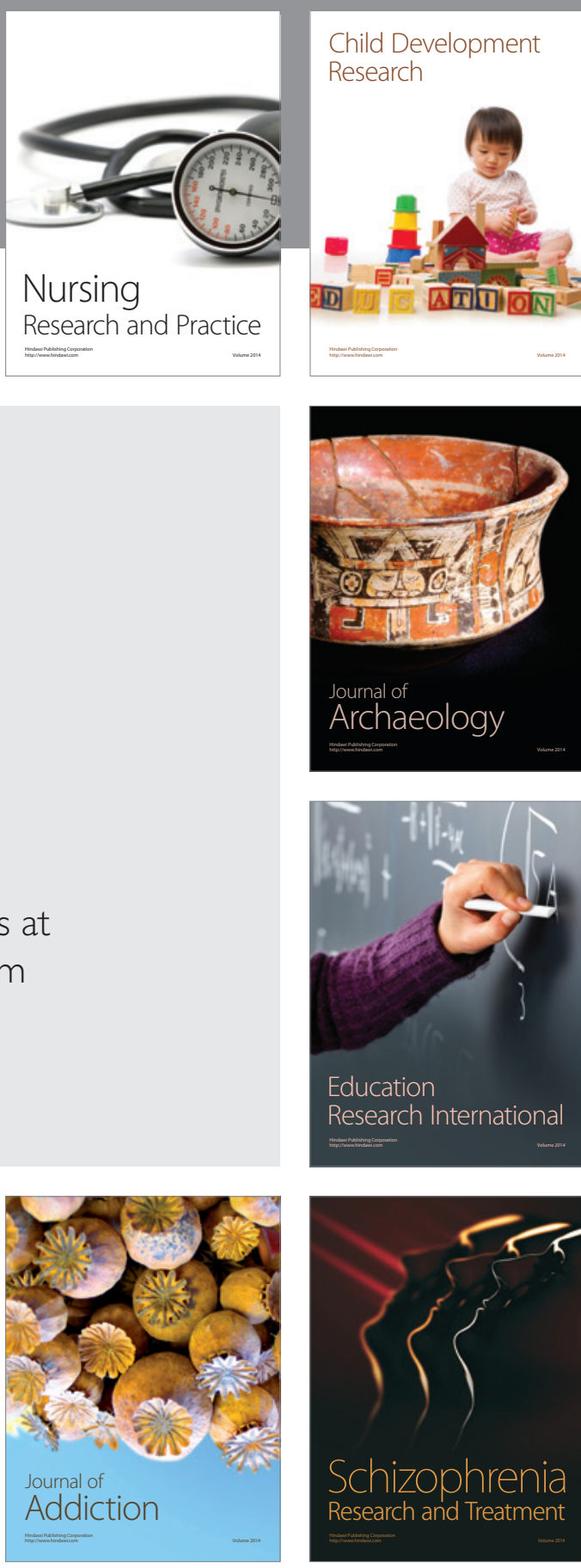

(D)
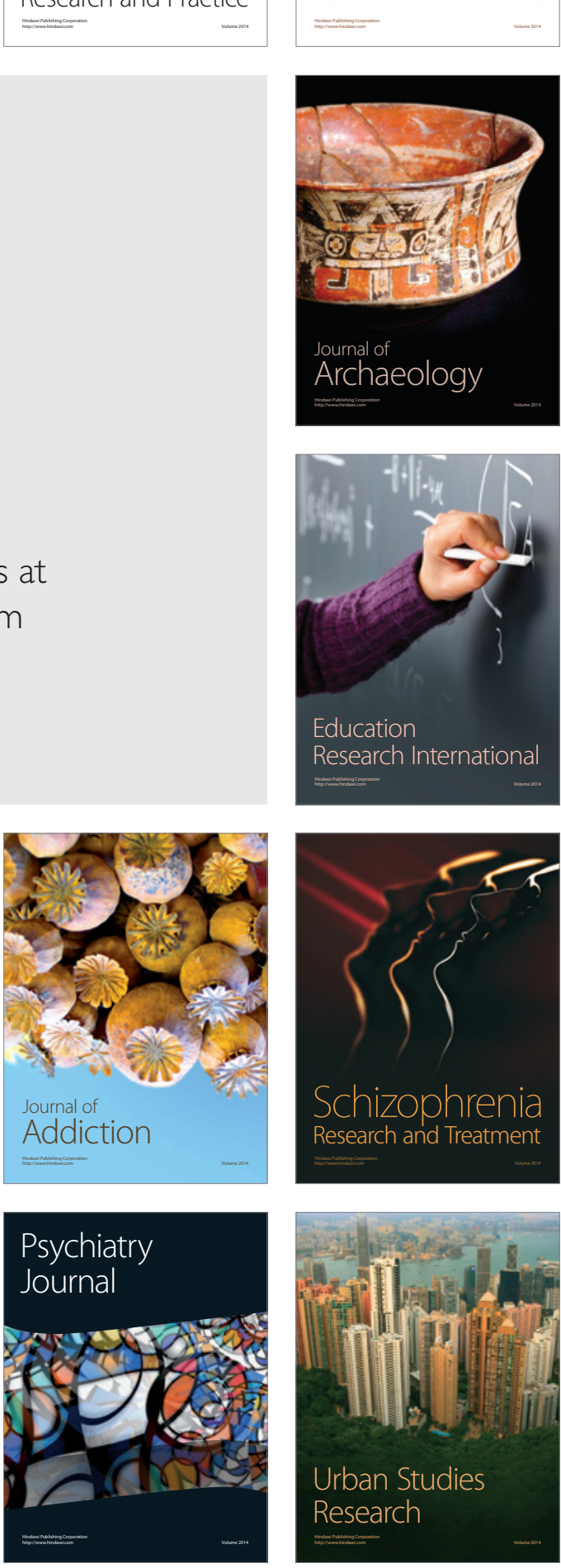